\title{
Associations of radiological features in Mycoplasma pneumoniae pneumonia
}

\author{
Qi Guo ${ }^{1}$, Hai-Yan Li², Yi-Ping Zhou ${ }^{1}$, Ming Li ${ }^{1}$, Xiao-Ke Chen ${ }^{1}$, Hong-Lin Peng ${ }^{1}$, Hai-Qiong Yư \\ Li-Hua Liang³, Qing-Zhou Zhao ${ }^{3}$, Mei Jiang ${ }^{4}$
}

\author{
'Department of Respiratory Medicine, Affiliated Futian Hospital, Guangdong Medical \\ College, Shenzhen, Guangdong, China \\ ²Department of Primary Care, Affiliated Futian Hospital, Guangdong Medical College, \\ Shenzhen, Guangdong, China \\ ${ }^{3}$ Department of Radiology, Affiliated Futian Hospital, Guangdong Medical College, \\ Shenzhen, Guangdong, China \\ ${ }^{4}$ Guangzhou Institute of Respiratory Diseases (State Key Laboratory of Respiratory \\ Diseases), First Affiliated Hospital, Guangzhou Medical University, Guangzhou, \\ Guangdong, China
}

Submitted: 23 June 2012

Accepted: 3 December 2012

Arch Med Sci 2014; 10, 4: 725-732

DOI: 10.5114 /aoms.2014.44863

Copyright (c) 2014 Termedia \& Banach

\begin{abstract}
Introduction: The associations of radiological features with clinical and laboratory findings in Mycoplasma pneumoniae infection are poorly understood. The purpose of this study was to assess the associations.

Material and methods: A retrospective cohort study of 1230 patients with community-acquired pneumonia was carried out between January 2005 and December 2009. The diagnosis of $M$. pneumoniae infection was made using the indirect microparticle agglutinin assay and enzyme-linked immunosorbent assay. Results: Females were more susceptible to M. pneumoniae infection. Groundglass opacification on radiographs was positively associated with $M$. pneumoniae-IgM titres (rank correlation coefficient $\left(r_{s}\right)=0.141, p=0.006$ ). The left upper lobe was more susceptible to infection with $M$. pneumoniae compared with other pathogens. More increases in the risk of multilobar opacities were found among older or male patients with M. pneumoniae pneumonia (odds ratio, 1.065, 3.279; 95\% confidence interval, 1.041-1.089, 1.812-5.934; $p<0.001, p<0.001$; respectively). Patients with $M$. pneumoniae pneumonia showing multilobar opacities or consolidation had a significantly longer hospital length of stay $\left(r_{\mathrm{s}}=0.111, r_{\mathrm{s}}=0.275 ; p=0.033\right.$, $p<0.001$; respectively), incurring significantly higher costs $\left(r_{\mathrm{s}}=0.119, r_{\mathrm{s}}=\right.$ $0.200 ; p=0.022, p<0.001$; respectively).

Conclusions: Our study highlighted female susceptibility to $M$. pneumoniae pneumonia and the association of ground-glass opacification with higher M. pneumoniae-lgM titres. The left upper lobe might be more susceptible to M. pneumoniae infection. Older or male patients with M. pneumoniae pneumonia were more likely to show multilobar opacities. Multilobar opacities and consolidation were positively associated with hospital length of stay and costs.
\end{abstract}

Key words: community-acquired pneumonia, Mycoplasma pneumoniae, radiological features, association.

\section{Introduction}

Community-acquired pneumonia (CAP) is a common infection. Despite substantial progress in therapeutic options, the mortality remains

\author{
Corresponding author: \\ Qi Guo MD, PhD \\ Department \\ of Respiratory Medicine \\ Affiliated Futian Hospital, \\ Guangdong Medical College \\ Shennan middle Road \\ No. 3025 \\ Shenzhen, Guangdong, \\ China, 518033 \\ Phone: +86 13923714808 \\ Fax: +86 75583986387 \\ E-mail: qiguo007@sina.com
}


unacceptably high [1], as high as $58 \%$ in patients with severe CAP admitted to the intensive care unit [2]. Mycoplasma pneumoniae is an important causative organism of CAP, accounting for $10-30 \%$ of all cases [3-5]. The frequency of CAP due to M. pneumoniae was $20.7 \%$ in China from December 2003 to November 2004 [6]. Patients infected with M. pneumoniae constitute about $29 \%$ of ambulatory adult patients with CAP and about $20 \%$ of hospitalized low-risk CAP patients in China [7, 8]. Three-four percent of patients infected with M. pneumoniae are known to develop a severe life-threatening pneumonia with respiratory failure and acute respiratory distress syndrome [9].

Although characteristic computed tomographic (CT) features of $M$. pneumoniae pneumonia have been described [10], radiological findings are not particularly specific. The disease is most frequently characterized by bronchopneumonic or interstitial infiltrates usually in the lower lobe, unilaterally and centrally dense [11].

Manifestations of infectious diseases may be influenced by both the pathogen and the host. In the case of $M$. pneumoniae pneumonia, mechanistic correlates of disease severity are poorly characterised. Generally, evidence from research on humans and animal models suggests that the immune system may on the one hand limit disease through the enhancement of host defence mechanisms but on the other hand exacerbate organ damage and systemic disease manifestations. Thus, the more vigorous the cell-mediated immune response and cytokine stimulation, the more severe the clinical illness and pulmonary injury [4]. Consequently, it might be expected that radiological signs of pulmonary lesions in M. pneumoniae pneumonia might vary according to the demographics of the patients and clinical and laboratory findings. To our knowledge, however, these associations have so far been only partly addressed by a few studies [10, 12-15].

In the present study, adult patients with CAP were selected to investigate the associations of radiological features with clinical and laboratory findings in a Chinese affiliated hospital.

\section{Material and methods}

\section{Design and setting}

A retrospective cohort study was performed of 1230 adult patients who met the criteria for CAP presenting to the Department of Respiratory Medicine in a Chinese affiliated hospital of a medical college from $1^{\text {st }}$ January 2005 to $31^{\text {st }}$ December 2009, thus including five autumn-winter seasons.

\section{Criteria for enrolment}

The CAP was defined as an acute infection of the pulmonary parenchyma associated with an acute infiltrate on the chest radiograph with two or more symptoms including fever $\left(>38^{\circ} \mathrm{C}\right)$, hypothermia $\left(<36^{\circ} \mathrm{C}\right)$, rigors, sweats, new cough or change in colour of respiratory secretions, chest discomfort or dyspnoea [16].

Acute $M$. pneumonia infection was defined as a fourfold or greater increase in specific IgM and/ or IgG titres between acute and convalescent phase serum samples. The CAP due only to acute M. pneumonia infection was defined as M. pneumoniae pneumonia. However, CAP caused by other pathogens and not having a fourfold or greater increase in specific lgM and/or IgG titres was defined as other pneumonias. Mixed pneumonia (atypical + bacteria) was discarded. Patients $<18$ years of age, any who had been hospitalized during the 28 days preceding the study, and any who had severe immunosuppression (e.g., patients with neutropenia after chemotherapy or bone marrow transplantation, patients with drug-induced immunosuppression as a result of solid-organ transplantation or corticosteroid or cytotoxic therapy, and patients with HIV-related disorders) [17], active tuberculosis, or end-stage diseases, or who had a written "do not resuscitate" order were excluded. We also excluded patients with pulmonary emphysema, idiopathic interstitial pneumonia and malignant diseases. In such patients it is difficult to distinguish bronchopneumonia from lobar pneumonia and unifocal from multifocal disease. There were no patients with concurrent infectious disease.

We used recent definitions of radiological abnormalities as follows [18]:

Ground-glass opacity: On chest radiographs, it appears as an area of hazy increased lung opacity, usually extensive, within which margins of pulmonary vessels may be indistinct. On CT scans, it appears as hazy increased opacity of lung, with preservation of bronchial and vascular margins.

Consolidation: It appears as a homogeneous increase in pulmonary parenchymal attenuation that obscures the margins of vessels and airway walls. An air bronchogram may be present.

\section{Grouping}

One thousand two hundred and forty-five patients were selected and then 15 cases were excluded from this investigation due to exclusion criteria. A total of 1230 patients were accepted for the study. Three hundred and seventy-two patients with $M$. pneumoniae-associated pneumonia were included in one group and compared with $858 \mathrm{pa}$ tients caused by infection due to other pathogens. Patients with ground-glass opacity were further designated as the bronchopneumonia subgroup and those with consolidation as the lobar pneumonia subgroup. Patients were also further subgrouped according to lobar involvement as follows: 


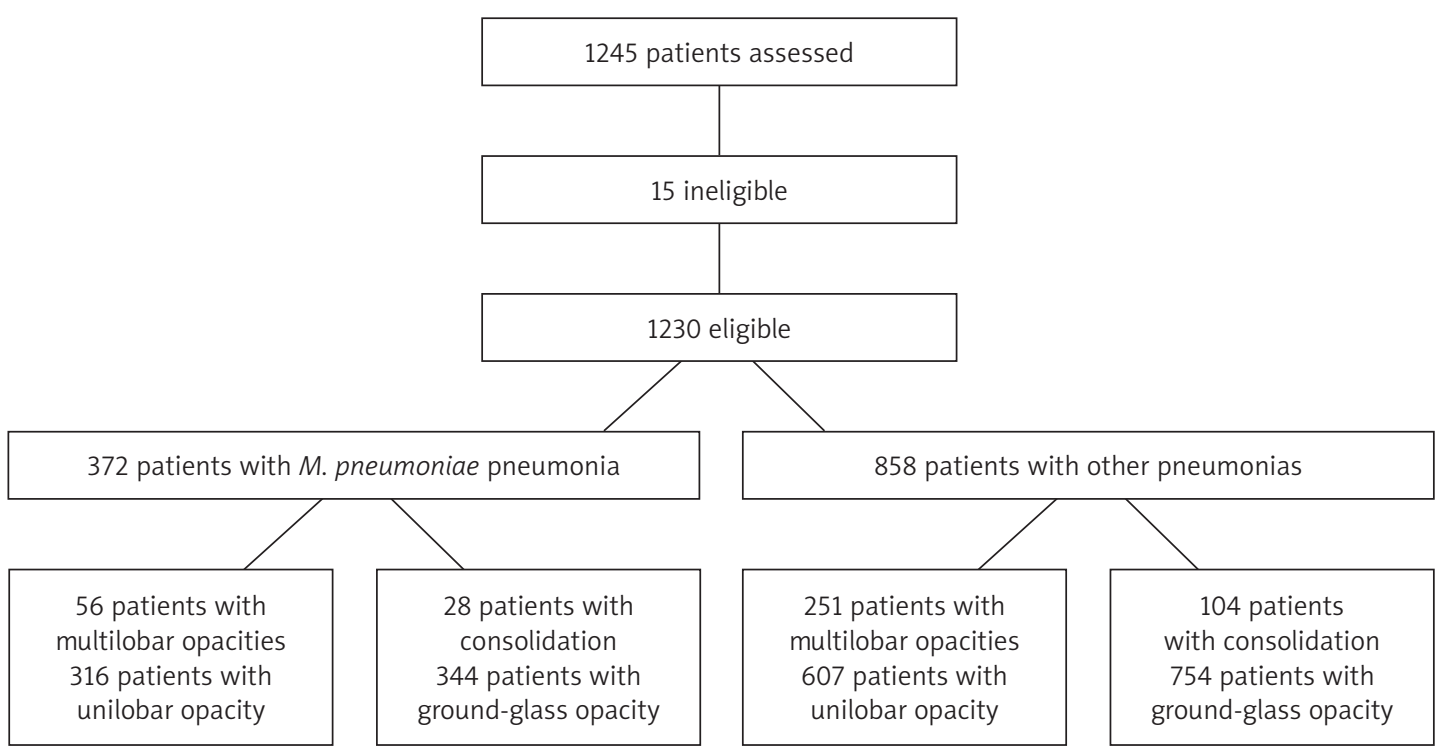

Figure 1. Study Flow Chart

the unilobar opacity subgroup and the multilobar opacities subgroup (Figure 1).

\section{Data collection}

Clinical and diagnostic data and radiological features were collected from medical records following discharge. For the detection of M. pneumoniae specific IgM antibodies, the indirect microparticle agglutinin assay (Serodia-Myco II, Fujirebio, Japan, positive threshold value $\geq 1: 40$ ) was used according to the manufacturer's instructions. Mycoplasma pneumoniae specific IgG antibodies were measured by SERION ELISA (enzyme-linked immunosorbent assay: Friedrich-Bergius-Ring 19, D-97076 Würzburg, Germany, positive threshold > $30 \mathrm{U} / \mathrm{ml}$ ). We analyzed $M$. pneumoniae-lgM titres as continuous variables after natural logarithmic transformation to normalize their distribution. CURB-65 (confusion, urea, respiratory rate, blood pressure, and age) scores at admission and sequential organ failure assessment (SOFA) scores at $72 \mathrm{~h}$ after commencing therapy were calculated. We also recorded total hospital costs, including pharmacy, radiology, and laboratory costs (including tests of blood, urine and stool, arterial blood gas, and bacterial cultures), examination costs (including lung function tests, electrocardiogram, and ultrasound), medical staff fees (including costs for physicians and nurses), bed or room fees, and other costs such as nebulization and oxygen therapy. Costs of dealing with multiple co-morbidities were excluded. All the patients had chest radiographs and CT scans. The frontal and lateral chest radiographic findings and CT scan images were reviewed and classified independently by two senior radiologists (LH Liang and QZ Zhao) and two senior physicians (YP Zhou and $\mathrm{M} \mathrm{Li}$ ) to ensure accuracy. Missing laboratory findings (most common in patients with less severe illness) were assumed to be normal [19]. The principal investigator reviewed every form and medical record to ensure accuracy. The study was approved by our Institutional Review Board (Review Board of Guangdong Medical College). No informed consent was required because the data were already in existence.

\section{Statistical analysis}

All statistical analyses were performed with Statistical Package for the Social Science for Windows version 13.0 (SPSS, Chicago, IL, USA). Categorical variables and continuous variables were reported as percentages and as the mean \pm standard deviation (SD), respectively. The $\chi^{2}$ test, unpaired Student's $t$-test, univariate logistic regression and Spearman rank correlation were employed. A $p$ value of $<0.05$ was considered statistically significant.

\section{Results}

\section{Baseline characteristics of patients}

Characteristics of the patients are presented in Table I. Patients infected with M. pneumoniae were younger, more likely to be female, and more likely to have a less severe condition compared with such patients infected with other pathogens. Mycoplasma pneumoniae was an important causative organism of CAP in the current study, accounting for $30.2 \%$ of all cases. All patients underwent diagnostic tests for the detection of the etiologic agent of pneumonia, but the aetiologies were detected in only $21.7 \%$ of the patients infected with other pathogens. Hence, the data were not shown. 
Table I. Baseline characteristics of the patients (mean \pm SD, $n=1230$ )

\begin{tabular}{|lcccc|}
\hline Variable & $\begin{array}{c}\text { M. pneumoniae } \\
\text { pneumonia group }\end{array}$ & $\begin{array}{c}\text { Other } \\
\text { pneumonias group }\end{array}$ & $t$ or $\chi^{2}$ value & Value of $p$ \\
\hline Age [years] & $31.3 \pm 11.6$ & $50.4 \pm 22.4$ & 15.702 & $<0.001$ \\
\hline Age $\geq 65$ years [\%] & 2.2 & 31.8 & 132.151 & $<0.001$ \\
\hline Male sex [\%] & 39.8 & 51.0 & 13.717 & $<0.001$ \\
\hline Confusion [\%] & 0 & 2.1 & 7.963 & 0.005 \\
\hline Body temperature [ $\left.{ }^{\circ} \mathrm{C}\right]$ & $37.5 \pm 1.1$ & $37.5 \pm 1.1$ & 0.281 & 0.779 \\
\hline Respiratory rate [breaths/min] & $19.7 \pm 2.3$ & $20.6 \pm 2.9$ & 5.053 & $<0.001$ \\
\hline Low blood pressure* [\%] & 15.1 & 14.2 & 0.171 & 0.679 \\
\hline WBC count [10\%/l] & $7.46 \pm 3.65$ & $8.83 \pm 4.42$ & 5.353 & $<0.001$ \\
\hline PaO ${ }_{2}$ /FiO $2 \leq 250$ mm Hg [\%] & 0 & 3.6 & 13.914 & $<0.001$ \\
\hline Serum BUN $\geq 7$ mmol/l [\%] & 0 & 7.5 & 29.413 & $<0.001$ \\
\hline Platelet count [10\%/l] & $245.69 \pm 96.52$ & $226.57 \pm 89.34$ & 3.469 & 0.001 \\
\hline CURB-65 score: 0-1-2-3-4 [\%] & $82.8-17.2-0-0-0$ & $53.8-35.4-9.4-1.1-0.2$ & 107.118 & $<0.001$ \\
\hline Multilobar opacities, $n$ (\%) & $15.1(56)$ & $29.3(251)$ & 29.295 & $<0.001$ \\
\hline Consolidation, $n$ (\%) & $7.5(28)$ & $12.1(104)$ & 5.838 & 0.016 \\
\hline
\end{tabular}

*Systolic < $90 \mathrm{~mm} \mathrm{Hg}$; or diastolic $\leq 60 \mathrm{~mm} \mathrm{Hg}, W B C$ - white blood cell, $\mathrm{PaO} / \mathrm{FiO}_{2}$ - arterial oxygen pressure/fraction inspired oxygen, BUN - blood urea nitrogen, CURB-65 - confusion, urea, respiratory rate, blood pressure, and age

\section{Mycoplasma pneumoniae-IgM titres}

The percentages of patients with $M$. pneumoniae-IgM titres of $1: 80,1: 160,1: 320,1: 640$, $1: 1280,1: 2560$ and $1: 5120$ in acute phase sera in the $M$. pneumoniae pneumonia group were $26.9,21.5,11.8,11.8,20.4,6.5$ and 1.1 , respectively. Patients with ground-glass opacification had higher M. pneumoniae-IgM titres compared with such patients with consolidation $(1: 2.524$ $\pm 1: 0.521$ vs. $1: 2.243 \pm 1: 0.414, t=2.789, p=$ 0.006 ), but there was no significant difference in the titres between patients with a unilobar opacity and those with multilobar opacities $(1: 2.515$ $\pm 1: 0.515$ vs. $1: 2.436 \pm 1: 0.539, t=1.024, p=$ $0.309)$. Ground-glass opacity showed a significant positive relationship with $M$. pneumoniae-lgM titres (rank correlation coefficient $\left(r_{s}\right)=0.141, p=$ 0.006). Mycoplasma pneumonia specific IgM titres in convalescent sera and $M$. pneumonia specific IgG titres were not shown.

\section{Distribution of opacities}

Distribution of opacities amongst the lobes is shown in Figure 2. A significant difference was observed between the two groups $\left(\chi^{2}=110.824\right.$, $p<0.001)$. Compared with other lobes, the left up-

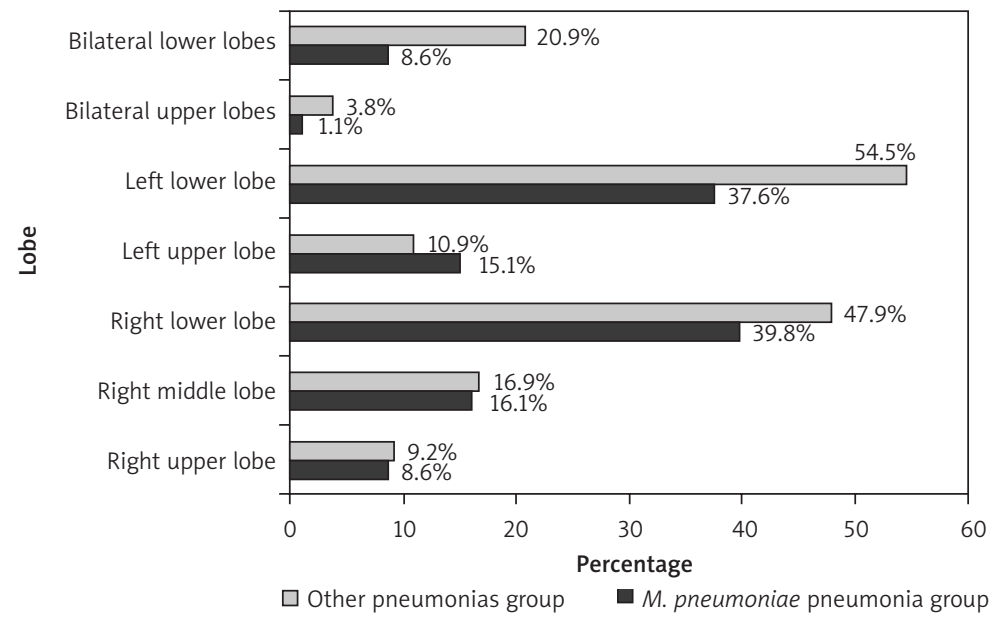

Figure 2. Distribution of opacities amongst the lobes 
per lobe appeared more susceptible to infection with $M$. pneumoniae than with other pathogens $\left(\chi^{2}=4.437, p=0.035\right)$.

\section{Radiological features according to demographic characteristics}

Associations of lobar involvement and parenchymal opacification with patients' demographic characteristics are depicted in Tables II and III, respectively. Comparisons between the M. pneumoniae pneumonia group and the other pneumonias group are listed in columns, whereas comparisons between the subgroups are shown in rows. More increases in the risk of multilobar opacities were found among older patients than younger patients in both the M. pneumoniae pneumonia group and the oth- er pneumonias group (odds ratio (OR), 1.065, 1.029; $95 \%$ confidence interval (CI), 1.041-1.089, $1.022-1.035 ; p<0.001, p<0.001$; respectively). Older patients with $M$. pneumoniae pneumonia also showed a trend towards lower susceptibility to lobar pneumonia than younger patients (OR $=0.971 ; 95 \% \mathrm{Cl}$ : 0.932-1.011; $p=0.155$ ), whereas older patients with other pneumonias had a decreased OR for lobar pneumonia compared with the younger patients $(\mathrm{OR}=0.989$; $95 \% \mathrm{Cl}: 0.980-0.997 ; p=0.009)$. An increased OR for multilobar opacities was observed in male patients with M. pneumoniae pneumonia compared with the female patients $(\mathrm{OR}=3.279$; 95\% Cl: 1.812-5.934; $p<0.001$ ), whereas with other pathogens this was decreased $(\mathrm{OR}=0.744$; 95\% Cl: 0.570-0.973; $p=0.030$ ).

Table II. Associations of lobar involvement with clinical and laboratory characteristics (mean $\pm S D, n=1230$ )

\begin{tabular}{|c|c|c|c|c|c|}
\hline Variable & Group & $\begin{array}{c}\text { Multilobar opacities } \\
\text { subgroup }\end{array}$ & $\begin{array}{l}\text { Unilobar opacity } \\
\text { subgroup }\end{array}$ & $t$ or $\chi^{2}$ value & Value of $p$ \\
\hline \multirow[t]{4}{*}{ Age [years] } & M. pneumoniae pneumonia & $39.9 \pm 15.2$ & $29.8 \pm 10.1$ & 6.349 & $<0.001$ \\
\hline & Other pneumonias & $60.2 \pm 23.2$ & $46.3 \pm 20.8$ & 9.485 & $<0.001$ \\
\hline & $t$ & 6.298 & 13.495 & & \\
\hline & $p$ & $<0.001$ & $<0.001$ & & \\
\hline \multirow[t]{4}{*}{ Male sex [\%] } & M. pneumoniae pneumonia & 64.3 & 35.4 & 16.519 & $<0.001$ \\
\hline & Other pneumonias & 45.8 & 53.1 & 4.695 & 0.030 \\
\hline & $\chi^{2}$ & 6.509 & 27.697 & & \\
\hline & $p$ & 0.011 & $<0.001$ & & \\
\hline \multirow{4}{*}{$\begin{array}{l}\text { CURB-65 } \\
\text { score }\end{array}$} & M. pneumoniae pneumonia & $0.29 \pm 0.46$ & $0.15 \pm 0.36$ & 2.459 & 0.014 \\
\hline & Other pneumonias & $0.90 \pm 0.85$ & $0.45 \pm 0.62$ & 9.523 & $<0.001$ \\
\hline & $t$ & 5.312 & 8.076 & & \\
\hline & $p$ & $<0.001$ & $<0.001$ & & \\
\hline \multirow[t]{4}{*}{ SOFA score } & M. pneumoniae pneumonia & $0.43 \pm 0.0 .74$ & $0.23 \pm 0.53$ & 2.459 & 0.014 \\
\hline & Other pneumonias & $1.20 \pm 1.59$ & $0.54 \pm 1.08$ & 7.762 & $<0.001$ \\
\hline & $t$ & 3.574 & 4.915 & & \\
\hline & $p$ & $<0.001$ & $<0.001$ & & \\
\hline \multirow{4}{*}{$\begin{array}{l}\text { Hospital LOS } \\
\text { [days] }\end{array}$} & M. pneumoniae pneumonia & $10.4 \pm 4.5$ & $9.1 \pm 3.0$ & 2.601 & 0.010 \\
\hline & Other pneumonias & $11.9 \pm 10.3$ & $9.4 \pm 4.4$ & 5.594 & $<0.001$ \\
\hline & $t$ & 1.126 & 1.514 & & \\
\hline & $p$ & 0.261 & 0.131 & & \\
\hline \multirow[t]{4}{*}{ Costs [\$] } & M. pneumoniae pneumonia & $768.22 \pm 411.26$ & $623.47 \pm 270.69$ & 3.375 & 0.001 \\
\hline & Other pneumonias & $1204.35 \pm 1323.26$ & $703.60 \pm 443.74$ & 9.122 & $<0.001$ \\
\hline & $t$ & 2.366 & 2.564 & & \\
\hline & $p$ & 0.019 & 0.010 & & \\
\hline
\end{tabular}

CURB-65 - confusion, urea, respiratory rate, blood pressure, and age, SOFA - sequential organ failure assessment, LOS - length of stay, $\$-$ US dollars 
Table III. Associations of parenchymal opacification with clinical and laboratory characteristics (mean \pm SD, $n=1230$ )

\begin{tabular}{|c|c|c|c|c|c|}
\hline Variable & Group & $\begin{array}{l}\text { Lobar pneumonia } \\
\text { subgroup }\end{array}$ & $\begin{array}{l}\text { Bronchopneumo- } \\
\text { nia subgroup }\end{array}$ & $t$ or $\chi^{2}$ value & Value of $p$ \\
\hline \multirow[t]{4}{*}{ Age [years] } & M. pneumoniae pneumonia & $28.3 \pm 8.1$ & $31.6 \pm 11.8$ & 1.966 & 0.057 \\
\hline & Other pneumonias & $43.8 \pm 19.7$ & $51.1 \pm 22.6$ & 3.360 & 0.001 \\
\hline & $t$ & 4.340 & 15.275 & & \\
\hline & $p$ & $<0.001$ & $<0.001$ & & \\
\hline \multirow[t]{4}{*}{ Male sex [\%] } & M. pneumoniae pneumonia & 28.6 & 40.7 & 1.589 & 0.207 \\
\hline & Other pneumonias & 49.2 & 51.2 & 0.178 & 0.915 \\
\hline & $\chi^{2}$ & 3.934 & 11.049 & & \\
\hline & $p$ & 0.047 & 0.001 & & \\
\hline \multirow{4}{*}{$\begin{array}{l}\text { CURB-65 } \\
\text { score }\end{array}$} & M. pneumoniae pneumonia & $0.29 \pm 0.46$ & $0.16 \pm 0.37$ & 1.659 & 0.098 \\
\hline & Other pneumonias & $0.56 \pm 0.77$ & $0.58 \pm 0.72$ & 0.329 & 0.742 \\
\hline & $t$ & 2.057 & 10.397 & & \\
\hline & $p$ & 0.041 & $<0.001$ & & \\
\hline \multirow[t]{4}{*}{ SOFA score } & M. pneumoniae pneumonia & $0.14 \pm 0.36$ & $0.27 \pm 0.58$ & 1.119 & 0.264 \\
\hline & Other pneumonias & $0.64 \pm 1.34$ & $0.75 \pm 1.28$ & 0.823 & 0.412 \\
\hline & $t$ & 3.539 & 6.741 & & \\
\hline & $p$ & 0.001 & $<0.001$ & & \\
\hline \multirow{4}{*}{$\begin{array}{l}\text { Hospital LOS } \\
\text { [days] }\end{array}$} & M. pneumoniae pneumonia & $14.1 \pm 4.4$ & $9.5 \pm 3.6$ & 5.409 & $<0.001$ \\
\hline & Other pneumonias & $14.4 \pm 13.3$ & $9.6 \pm 5.2$ & 7.334 & $<0.001$ \\
\hline & $t$ & 0.100 & 0.250 & & \\
\hline & $p$ & 0.920 & 0.803 & & \\
\hline \multirow[t]{4}{*}{ Costs [\$] } & M. pneumoniae pneumonia & $823.44 \pm 243.93$ & $630.76 \pm 299.72$ & 3.944 & $<0.001$ \\
\hline & Other pneumonias & $1181.28 \pm 1311.15$ & $808.57 \pm 749.44$ & 4.579 & $<0.001$ \\
\hline & $t$ & 2.569 & 4.001 & & \\
\hline & $p$ & 0.011 & $<0.001$ & & \\
\hline
\end{tabular}

CURB-65 - confusion, urea, respiratory rate, blood pressure, and age, SOFA - sequential organ failure assessment, LOS - length of stay, S- US dollars

\section{Associations of lobar involvements with clinical and laboratory characteristics}

Associations of lobar involvements with clinical and laboratory characteristics are shown in Table II. Multilobar opacities had significant positive relationships with CURB-65 scores in both the M. pneumoniae pneumonia group and the other pneumonias group $\left(r_{s}=0.127, r_{s}=0.262 ; p=0.014\right.$, $p<0.001$; respectively). Multilobar opacities were associated with longer hospital LOS in both the M. pneumoniae pneumonia group and the other pneumonias group $\left(r_{\mathrm{s}}=0.111, r_{\mathrm{s}}=0.083\right.$; $p=0.033, p=0.008$; respectively), incurring significantly higher costs $\left(r_{\mathrm{s}}=0.119, r_{\mathrm{s}}=0.201 ; p=\right.$ $0.022, p<0.001$; respectively).

\section{Associations of parenchymal opacification with clinical and laboratory characteristics}

Associations of parenchymal opacification with clinical and laboratory characteristics are shown in Table III. Consolidation had significant positive relationships with hospital LOS in both the M. pneumo- niae pneumonia group and the other pneumonias group $\left(r_{\mathrm{s}}=0.275, r_{\mathrm{s}}=0.192 ; p<0.001, p<0.001\right.$; respectively), as it did with costs despite no association with the severity of the disease $\left(r_{\mathrm{s}}=0.200\right.$, $r_{s}=0.151 ; p<0.001, p<0.001$; respectively).

\section{Discussion}

Our findings show that females were more susceptible to $M$. pneumoniae infection, that ground-glass opacification had a positive association with M. pneumoniae-lgM titres, that the left upper lobe was more susceptible to infection with M. pneumoniae, that older or male patients with M. pneumoniae pneumonia were more likely to show multilobar opacities, and that patients with M. pneumoniae pneumonia showing multilobar opacities or consolidation had a significantly longer hospital LOS and higher costs.

Radiographic manifestations of $M$. pneumoniae pneumonia can be extremely variable and mimic a wide variety of lung diseases. The inflammatory response elicited by $M$. pneumoniae causes interstitial mononuclear cell inflammation that may be 
manifested radiographically as diffuse, reticular infiltrates of bronchopneumonia in the perihilar regions or lower lobes, usually with a unilateral distribution, and hilar adenopathy [4]. In the current study, the common radiographic features of M. pneumoniae pneumonia on chest radiographs and CT scans were ground-glass opacification, unilobar opacification, or lower lobe involvement as previously described by Mansel et al. [11] and Reittner et al. [20]. Mycoplasma pneumoniae is an extracellular pathogen, the survival of which depends on adherence to the respiratory epithelium, and this fixation to ciliary membranes is conducted primarily by interactive adhesion and accessory proteins. The major adhesion protein is $\mathrm{P} 1 \mathrm{ad}$ hesin. Cytoadhesion protects $M$. pneumoniae from mucociliary clearance [21]. Hydrogen peroxide and superoxide radicals synthesized by $M$. pneumoniae act in concert with endogenous toxic oxygen molecules generated by host cells to induce oxidative stress in the respiratory epithelium [4]. Attachment of the microorganism to epithelial cilia is responsible for bronchial wall thickening [21]. Therefore, this attachment might be envisaged to interpret higher frequency of the presentation of ground-glass opacification in $M$. pneumoniae pneumonia compared with other pneumonias and the association of ground-glass opacification with higher M. pneumoniae-lgM titres. Our finding that lobar involvement did not associate with IgM titres is not congruent with what Waites et al. [4] observed (the cold agglutinin response often correlates directly with the severity of pulmonary involvement). On the basis of the current knowledge, we can envisage no definite mechanisms to account for the apparent increase in susceptibility of females to M. pneumoniae infection. As mycoplasma occurs in epidemic cycles, there may be local epidemiologic factors that may have played a role in the female preponderance to mycoplasma infection. Similarly, there is little information to determine the possible reasons why the left upper lobe was more susceptible to infection with M. pneumoniae than with other pathogens and it requires further epidemiological observations. Hence, further studies are warranted.

The frequency of consolidation in association with $M$. pneumoniae infection appears to vary greatly between studies. One study reported that consolidation was infrequent in adult patients in Japan [10], Hsieh et al. [12] reported a higher frequency of this pattern (38\%) in paediatric patients in Taiwan, whereas Hosker et al. [13] reported that $95 \%$ of paediatric patients presented with this pattern in Hong Kong and Defilippi et al. [14] showed that consolidation was the commonest finding in children with infection due to M. pneumoniae in Genoa. Bilateral involvement may occur in about $20 \%$ of cases [22], but it was observed in only $9.7 \%$ of the patients in the current study.

The factors determining disease manifestations and severity in $M$. pneumoniae infection are only partly understood. Few studies have attempted a clinical comparison of the effects of M. pneumoniae infection according to age. Youn et al. [15] reported that paediatric patients with segmental/lobar pneumonia were older than those with bronchopneumonia in Korea. Defilippi et al. [14] also found that the rate of chest radiographic consolidation was higher in older children with M. pneumoniae infection. There was a significantly increased OR for multilobar opacities in older adult patients and younger adult patients showed a trend toward susceptibility to lobar pneumonia in the current study. These may reflect the fact that the immune response and/or the mucociliary clearance of $M$. pneumoniae are less active in older adults and younger children, conferring a risk of more severe disease. On the basis of the above-mentioned rationale, it is difficult to interpret the positive association of multilobar opacities with male patients suffering from $M$. pneumoniae pneumonia in the current study. It warrants further study.

In the current study, the positive association of multilobar opacities, but not consolidation, with severity of the disease is consistent with the common understanding that the presentation of multilobar opacities is one of nine minor criteria for severe CAP [1]. There have been few previous studies about the associations of radiological features with hospital LOS and costs in patients with pneumonia due to $M$. pneumoniae. The length of stay depends on the time needed to reach clinical stability, which is significantly influenced by the severity of disease [23]. Bilateral or multilobar opacities on chest radiographs of low-risk CAP patients were not significantly associated with higher total costs [8], but we found that multilobar opacities were positively associated with hospital LOS and costs in both groups, as was consolidation despite no association with the severity of the disease, which might have implications for the management of $M$. pneumoniae pneumonia and other pathogens pneumonia and health economics.

A few laboratory findings were inevitably missed due to retrospective data collection. This phenomenon is most common in patients with less severe illness. Missing values were assumed to be normal in the current study. This strategy is widely used in the clinical application of prediction rules and reflects the methods used in the original derivation and validation of the Pneumonia Severity Index [19]. Even so, some of them might be abnormal. Therefore, the assumption might result in the alterations of statistical power and even statistical results and might give rise to misleading 
conclusions. Hence, further prospective validation of our findings is warranted.

Several limitations of this study deserve comment. First, the numbers of patients with consolidation or multilobar opacities were relatively small despite this being a cohort study. Had the number been larger, perhaps the results might have been more robust. The best method to detect acute M. pneumoniae infection is a polymerase chain reaction assay. Therefore, a few patients infected with $M$. pneumoniae might be excluded because of the absence of IgM antibodies in the early stage. It was not multicentre. A further weakness of this study was its retrospective design.

In conclusion, females were more susceptible to $M$. pneumoniae pneumonia. Ground-glass opacification was associated with higher M. pneumoniae-lgM titres. The left upper lobe might be more likely to be infected with $M$. pneumoniae compared with other pathogens. Older or male patients with $M$. pneumoniae pneumonia were more likely to show multilobar opacities. Multilobar opacities and consolidation were positively associated with hospital LOS and costs.

\section{Acknowledgments}

Qi Guo and Hai-Yan Li are joint first authors.

We are indebted to the nurses, further education physicians and postgraduates of the Department of Respiratory Medicine, and the staff of the Department of Medical Record for making contributions to this study.

We thank Chris J. Corrigan (King's College London) for assistance in English editing.

The abstract (No. 501) has been accepted for a Thematic Poster at the ERS (European Respiratory Society) Amsterdam 2011 Annual Congress (24-28 September).

The study was funded by the medical science and technology foundation of Guangdong province in 2010 (No. A2010553), the planned science and technology project of Shenzhen municipality in 2011 (No. 201102078), and the non-profit scientific research project of Futian district in 2011 (No. FTWS201120).

\section{References}

1. Mandell LA, Wunderink RG, Anzueto A, et al. Infectious Diseases Society of America/American Thoracic Society consensus guidelines on the management of community-acquired pneumonia in adults. Clin Infect Dis 2007; 44 (Suppl 2): S27-72.

2. Restrepo MI, Anzueto A. Severe community-acquired pneumonia. Infect Dis Clin North Am 2009; 23: 503-20.

3. Blasi F. Atypical pathogens and respiratory tract infections. Eur Respir J 2004; 24: 171-81.

4. Waites KB, Talkington DF. Mycoplasma pneumoniae and its role as a human pathogen. Clin Microbiol Rev 2004; 17: 697-728.
5. Atkinson TP, Balish MF, Waites KB. Epidemiology, clinical manifestations, pathogenesis and laboratory detection of Mycoplasma pneumoniae infections. FEMS Microbiol Rev 2008; 32: 956-73.

6. Liu YN, Chen MJ, Zhao TM, et al. A multicentre study on the pathogenic agents in 665 adult patients with community-acquired pneumonia in cities of China. Zhonghua Jie He He Hu Xi Za Zhi 2006; 29: 3-8.

7. Cao B, Ren LL, Zhao F, et al. Viral and Mycoplasma pneumoniae community-acquired pneumonia and novel clinical outcome evaluation in ambulatory adult patients in China. Eur J Clin Microbiol Infect Dis 2010; 29: 1443-8.

8. Zhou QT, He B, Zhu H. Potential for cost-savings in the care of hospitalized low-risk community-acquired pneumonia patients in China. Value Health 2009; 12: 40-6.

9. Koletsky RJ, Weinstein AJ. Fulminant Mycoplasma pneumoniae infection. Report of a fatal case, and review of the literature. Am Rev Respir Dis 1980; 122: 491-6.

10. Miyashita N, Sugiu T, Kawai Y, et al. Radiographic features of Mycoplasma pneumoniae pneumonia: differential diagnosis and performance timing. BMC Med Imaging 2009; 9: 7.

11. Mansel JK, Rosenow EC 3rd, Smith TF, Martin JW Jr. Mycoplasma pneumoniae pneumonia. Chest 1989; 95: 639-46.

12. Hsieh SC, Kuo YT, Chern MS, Chen CY, Chan WP, Yu C. Mycoplasma pneumonia: clinical and radiographic features in 39 children. Pediatr Int 2007; 49: 363-7.

13. Hosker HS, Tam JS, Chain CH, Lai CK. Mycoplasma pneumoniae infection in Hong Kong: clinical and epidemiological features during an epidemic. Respiration 1993; 60: 237-40.

14. Defilippi A, Silvestri M, Tacchella A, et al. Epidemiology and clinical features of Mycoplasma pneumoniae infection in children. Respir Med 2008; 102: 1762-8.

15. Youn YS, Lee KY, Hwang JY, et al. Difference of clinical features in childhood Mycoplasma pneumoniae pneumonia. BMC Pediatr 2010; 10: 48.

16. Phua J, See KC, Chan YH, et al. Validation and clinical implications of the IDSA/ATS minor criteria for severe community-acquired pneumonia. Thorax 2009; 64: 598-603.

17. Hilbert G, Gruson D, Vargas F, et al. Noninvasive ventilation in immunosuppressed patients with pulmonary infiltrates, fever, and acute respiratory failure. N Engl J Med 2001; 344: 481-7.

18. Hansell DM, Bankier AA, MacMahon H, McLoud TC, Müller NL, Remy J. Fleischner Society: glossary of terms for thoracic imaging. Radiology 2008; 246: 697-722.

19. Aujesky D, Auble TE, Yealy DM, et al. Prospective comparison of three validated prediction rules for prognosis in community-acquired pneumonia. Am J Med 2005; 118: 384-92.

20. Reittner P, Müller NL, Heyneman L, et al. Mycoplasma pneumoniae pneumonia: radiographic and high-resolution CT features in 28 patients. AJR Am J Roentgenol 2000; 174: 37-41.

21. Kashyap S, Sarkar M. Mycoplasma pneumonia: clinical features and management. Lung India 2010; 27: 75-85.

22. Ferwerda A, Moll HA, de Groot R. Respiratory tract infections by Mycoplasma pneumoniae in children: a review of diagnostic and therapeutic measures. Eur J Pediatr 2001; 160: 483-91.

23. Halm EA, Fine MJ, Marrie TJ, et al. Time to clinical stability in patients hospitalized with community-acquired pneumonia: implications for practice guidelines. JAMA 1998: 279: 1452-7. 